\title{
Detailed simulation of air-assisted spray atomization: effect of numerical scheme at intermediate Weber number
}

\author{
G. Tretola ${ }^{1 *}$, K. Vogiatzaki ${ }^{2}$, S. Navarro-Martinez ${ }^{1}$ \\ ${ }^{1}$ Department of Mechanical Engineering, Imperial College, London SW7 2AZ, UK \\ ${ }^{2}$ School of Engineering and Mathematics, University of Brighton, Brighton, BN2 4AT, UK \\ *Corresponding author: g.tretola16@imperial.ac.uk
}

\begin{abstract}
Numerical simulations are often used to understand spray atomisation and estimate the size of the liquid fragments. Several techniques (Level Set, Volume of Fluid, Smooth Particle Hydrodynamics, among others) exist to compute multiphase flows and potentially represent liquid-break-up. However, the complexity of the breakup process and the wide range of scales prevents the use of an unified approach to simulate the complete spray. Numerical techniques face different challenges depending on the spray characteristics. The incorrect representation of surface forces in capillary dominated flows, creates large parasitic currents that distort and in some cases destroy the interface. Methods that perform well in the capillary regime aim to capture the interface directly and the surface radius curvature is therefore larger than the mesh size. However, this creates large constrains on the mesh resolution and limits its applications to low Weber number flows, when there is no extensive atomization. Methods that simulate large Weber number flows (typical of industrial injectors) do not resolve the interface directly and the mesh is larger than the smallest radius of curvature. These models often have numerical or artificial diffusion that destroys small scale structures and alters the break-up. However, even at large Weber flows, the spray formation can be affected by errors due to the local imbalance between pressure and surface tension forces and interface curvature errors. Numerical schemes work around these problems by adjusting the amount of numerical diffusion of the scheme depending on the spray application. Intermediate Weber number sprays are well suited to study the performance of numerical methods as they exhibit hybrid behaviour between capillary flows and full atomization. In the present work an intermediate gas Weber of a laboratory air-blast atomiser is investigated using a volume of fluid approach. The amount of numerical diffusion is controlled by a compressive factor in the volume of fluid transport equation. The effect of the compressive term on spray atomization and droplet size distribution is explored. The results suggest that the optimal amount of diffusion depends on the local Weber number.
\end{abstract}

\section{Keywords}

VOF, OpenFOAM, Atomisation, intermediate Weber.

\section{Introduction}

Liquid atomization is a complex phenomenon present in many engineering processes. The atomization process depends on complex interactions between aerodynamic and capillary forces. Liquid structures are shed from the dense spray core, forming ligaments that pinch-off and form droplets. The droplet break-up pattern itself is also very complex, a droplet may break into few large droplets (for example: vibrational break-up) or a myriad of small droplets (bag break-up), among other. For detailed reviews regarding the physics of the atomisation, see [4] and [5].

The atomisation process involves a wide range of length scales: from the nozzle diameter to the smallest droplets. The smallest scale in atomisation is not still characterised and this size can be three orders of magnitude smaller than the large-scale diameter depending on the Weber number. In order to achieve efficient atomization, two strategies are often used: either the liquid is accelerated to high velocities in a near-quiescent gas, pressurised injection; or the liquid fuel is injected at low speed, surrounded by a coflow high-velocity gas, air-assisted injection. When multiphase flows are solved numerically, the different phases need to be identified. Two major approaches exist: one is the two-fluid model [1] where each phase is governed by an individual set of transport equations for mass, momentum, and total energy. These methods have been implemented either in a pure Eulerian way or most commonly in an Eulerian/Lagrangian framework. An alternative approach is the one-fluid model, where the two phases are treated as one, with a single mixture velocity but a variable density and viscosity. The amount of volume that each phase occupies in the mixture fluid results from a marker function (hereafter $\alpha$ ), also called a "color" function, which follows a simple advection transport equation. The popular volume of fluid (VOF) approach [6] belongs to this category; where the marker function is the fractional volume occupied by one fluid, in liquid-gas simulations often taken as the liquid. There are more approaches to model multiphase flows, however, a detailed review is out of the scope of this work and the reader is referred to [7].

VOF methods are widely spread and have been implemented in many commercial and open-source CFD software. In low Weber number flows, VOF-techniques aim to solve the interface directly as it is possible to have enough computational points within a radius of curvature. Methods in this regime use low-diffusion or compressive numerical schemes to transport the marker $\alpha$ and maintain a sharp interface. However, "classical" VOF requires an interface reconstruction to evaluate the interface curvature to compute surface forces. The accuracy of the reconstruction is based on the smoothness of the marker function and errors create parasitic currents that disturb the interface [14]. 
Numerical VOF methods at large Weber (typical of industrial injectors) do not have a completely resolved interface and they posses large numerical diffusion (as the interface is thickened over several cells). This "artificial" diffusion, destroys small scale structures and alters the break-up process. However, even at large Weber numbers, the local Weber can be relatively small and the spray formation affected by error in the local imbalance between pressure and capillary forces.

The present work aims to investigate the effect of the numerical scheme on the spray characteristics, mainly the droplet size distribution and spray core length. The test case is an air-assisted laboratory spray at intermediate Weber, where VOF-techniques will face challenges corresponding to both Weber regimes. By varying the "compression" of the numerical scheme (and therefore its numerical diffusion) the numerical scheme effects on atomisation can be shown.

The goal is to find a local parameter that can be used to adapt the numerical scheme to the local requirements.

The paper is organised as follows: First, the mathematical model for the VOF scheme and its implementation are presented. Followed by the results section; where the effects of the numerical scheme are presented through snapshots of the marker and the droplet size distributions.

\section{Mathematical model}

The incompressible one-fluid Navier-Stokes equations neglecting body forces together with the $\alpha$ transport equation are:

$$
\begin{aligned}
& \nabla \cdot \mathbf{u}=0 \\
& \frac{\partial \rho \mathbf{u}}{\partial t}+\nabla \cdot(\rho \mathbf{u u})=-\nabla p+\nabla \cdot \mathbf{T}+\rho \mathbf{f}_{\sigma} \\
& \frac{\partial \alpha}{\partial t}+\nabla \cdot(\mathbf{u} \alpha)=0
\end{aligned}
$$

where $\mathbf{u}$ represent the mixture velocity and the stress tensor is $\mathbf{T}=\mu\left[\nabla \mathbf{u}+(\nabla \mathbf{u})^{T}\right]$. The surface forces assuming a constant surface tension are

$$
\mathbf{f}_{\sigma}=\sigma \kappa \mathbf{n} \cdot \delta_{S}
$$

where the curvature of the surface is $\kappa=\nabla \cdot \mathbf{n}$ In the VOF method, the marker $\alpha$ is used to compute the surface normals $\mathbf{n}=\nabla \alpha$ and the surface force is implemented as a body force using the continuum surface force (CSF) model [8].

Ideally, the interface between the two phases should be massless since it represents a sharp discontinuity and $\alpha$ should be a discontinuous function which takes the value 0 for gas and 1 for liquid. However in a finite volume formulation, intermediate values of $\alpha$ exist in cells where the interface is present. In practice due to the numerical diffusion of Eqn.(3) intermediate value of $\alpha$ are present in a "thick" region around the interface. The physical properties are calculated as weighted averages based on $\alpha$, viz.

$$
\begin{gathered}
\rho=\alpha \rho_{l}+(1-\alpha) \rho_{g} \\
\mu=\alpha \mu_{l}+(1-\alpha) \mu_{g}
\end{gathered}
$$

Equation (3) can be modified by assuming that the mixture velocity can be defined as a weighted average of the phase velocities (see [10])

$$
\mathbf{u}=\alpha \mathbf{u}_{l}+(1-\alpha) \mathbf{u}_{g}
$$

Replacing the velocity by the above definition into the transport equation for $\alpha$ :

$$
\frac{\partial \alpha}{\partial t}+\nabla \cdot\left\{\left[\alpha \mathbf{u}_{l}+(1-\alpha) \mathbf{u}_{g}\right] \alpha\right\}=0
$$

From the definition of the relative velocity $\mathbf{u}_{r}=\mathbf{u}_{l}-\mathbf{u}_{g}$, is possible to isolate the gas velocity from the above equation, and rearranging as:

$$
\frac{\partial \alpha}{\partial t}+\nabla \cdot\left(\mathbf{u}_{l} \alpha\right)-\nabla \cdot\left[(1-\alpha) \alpha \mathbf{u}_{r}\right]=0
$$

The first two terms represent the transport of $\alpha$ in the liquid phase, which in incompressible flows is $0 \partial \alpha / \partial t+$ $\mathbf{u}_{l} \nabla \alpha=0$. Therefore,

$$
\nabla \cdot\left[(1-\alpha) \alpha \mathbf{u}_{r}\right]=0
$$

The term can therefore be added to Eqn. (3), obtaining

$$
\frac{\partial \alpha}{\partial t}+\nabla \cdot(\mathbf{u} \alpha)-\nabla \cdot\left[(1-\alpha) \alpha \mathbf{u}_{r}\right]=0
$$


The last term in the above equation is the compression contribution, which is active only at the interface region and has no physical meaning in the continuum formulation. However, in a discrete formulation it acts as an anti-diffusion term and provides a sharper interface compared to the classical VOF formulations, The model does not alter mass conservation of the scheme, however a closure is needed for the relative velocity, $\mathbf{u}_{r}$, as the phase velocities are not available in an one-fluid formulation.

\section{Numerical Implementation}

To solve Eqns. (1), (2) and (11) the unstructured finite volume solver interFoam [11] is used, which is implemented into OpenFOAM [9] The compression term is evaluated using the divergence theorem and the corresponding relative velocity at the cell faces $u_{r, f}=\mathbf{u}_{r, f} \cdot \mathbf{n}_{f}$ is modelled proportional to face normal flow velocity

$$
u_{r, f}=C_{\alpha} u_{f}^{*}
$$

where the subscript $f$ indicates the corresponding cell face. As reported and highlighted in [11], $u_{f}^{*}$ is not evaluated by face interpolation of the velocity, but is obtained from the conservative volume flux from the pressure-velocity coupling algorithm to maintain $\nabla \cdot \mathbf{u}=0$.

The model is based on the definition of relative velocity in Eqn. (7) and suggest an dependence of both $\alpha$ and flow across the face. The constant $C_{\alpha}$ controls the magnitude of the compression term and therefore the diffusion of the numerical scheme. $C_{\alpha}$ is a user-specified value, which in capillary flow is often case specific [12]. Small values of $C_{\alpha}$, provide moderate compression and numerically diffusive schemes (small relative velocity), while large compression, $C_{\alpha} \geq 1$ provide sharper interfaces.

\section{Results and discussion Numerical Set-Up}

The selected test-case is an air-assisted atomiser, presented and studied by [3] and [2]. The injector geometry consists of a straight jet of diameter $d_{1}=1.3335 \cdot 10^{-3} \mathrm{~m}$, where $\mathrm{n}$-dodecane flows. The pipe is surrounded by a co-flow of inner diameter $d_{2}=1.5875 \cdot 10^{-3} \mathrm{~m}$ and thickness $h=2.8575 \cdot 10^{-4} \mathrm{~m}$, with a faster nitrogen stream. The main properties with the Reynolds and Weber numbers are presented in Table 1. The gas-to-liquid momentum ratio is $M=2.53$, which suggest a length core of 3.9 jet diameters (based on experimental correlations [15]) The simplicity of the geometry is well suited for numerical modelling and validation. Desjardins et al. [3] performed Direct Numerical Simulation using an advanced level-set method of the same configuration, with a mesh of $512 \times 256 \times 256$ on a domain of $16 d_{1} \times 8 d_{1} \times 8 d_{1}$. The atomization DNS was performed on 1024 processors [3]. In the present work, the grid size is increased approximately three times in each direction respect to the base DNS, using a mesh of $180 \times 90 \times 90$ grid cells and the simulations were performed in a single workstation. The computational domain is the same as the DNS and a constant CFL number below 0.9 is used throughout the simulation. The code used is the VOF solver interFoam present in OpenFOAM 3.0. Due to the relative low Reynolds number at the tubes outlet, turbulence modelling is neglected.

Table 1. Liquid and gas properties and flow properties for the test case

\begin{tabular}{c|cccccc}
\hline & $\rho\left[\mathrm{kg} / \mathrm{m}^{3}\right]$ & $\mu[\mathrm{kg} / \mathrm{ms}]$ & $\sigma[\mathrm{N} / \mathrm{m}]$ & $U[\mathrm{~m} / \mathrm{s}]$ & $R e[-]$ & $W e[-]$ \\
\hline Liquid & 746 & $1.36 \cdot 10^{-3}$ & $2.535 \cdot 10^{-2}$ & 1.8 & 1336 & 127 \\
Gas & 1.25 & $1.718 \cdot 10^{-5}$ & $2.535 \cdot 10^{-2}$ & 69.89 & 1453 & 321
\end{tabular}

\section{Results}

Figure 1 shows the interface for three different values of the compression factor $C_{\alpha}: 0.1,1$ and 4 . The original DNS [3] showed the formation of the instabilities along the liquid core, which develop into liquid ligaments and then droplets. The present results show similar overall characteristics but with a large dependency of the compression ratio.
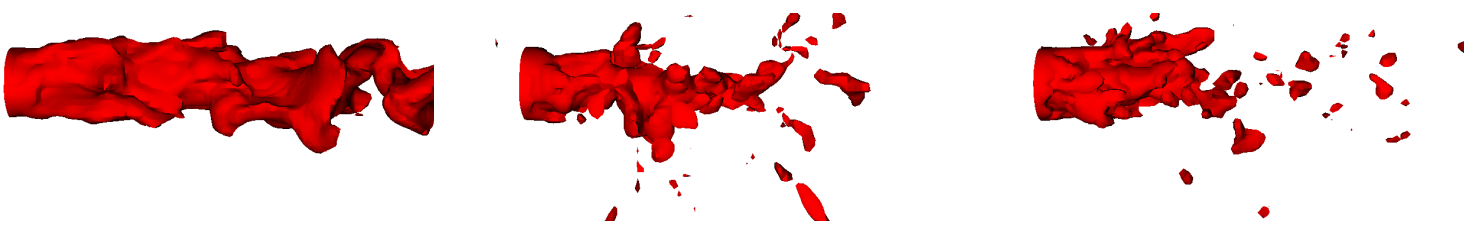

Figure 1. Iso-surface of $\alpha=0.05$ close to the injector. From left to right, $C_{\alpha}=0.01,1$ and 4 , respectively.

Figure 2, shows different iso-contours of $\alpha$ at different compression factors. The distance between the minimum and maximum iso-levels of $\alpha=0.01$ and 0.7 can be understood as a measure of the artificial thickness of the interface. 
The value of 0.7 to represent the core jet is arbitrary and in principle any value of $\alpha$ grater then 0.5 can be used. Nevertheless, in the present simulation the iso-levels greater than 0.5 are very close together (see Fig 2 ) and the precise choice of iso-contour does not affect the discussion."

In the break-up region, the results with the $C_{\alpha}=0.01$ shows an interface thickness of more than 10 cells. In this case, the liquid core does not break but follows a mixing profile typical of gaseous jets. Assuming the dense core is determined by the iso-line of $\alpha=0.7$, both $C_{\alpha}=1$ and $C_{\alpha}=4$ simulations have similar core lengths of $L_{c} / d_{1} \approx 4$, which agree well with empirical correlations $L_{c} / d_{1} \approx 6 / \sqrt{M}$. However, the structure after break-up is significantly different.

For $C_{\alpha}=0.01$, regions with low volume fraction are pushed towards the side, forming ligaments, while large volume fraction regions remain near the centre line of the injector. The results are that only a small fraction of liquid goes into ligaments therefore preventing the break-up. Large liquid structure detaches from the core carried on along the centre by the gas phase. At large compression factors, the thickness of the interface is reduced and dense ligaments form. Then these ligaments break into droplets.
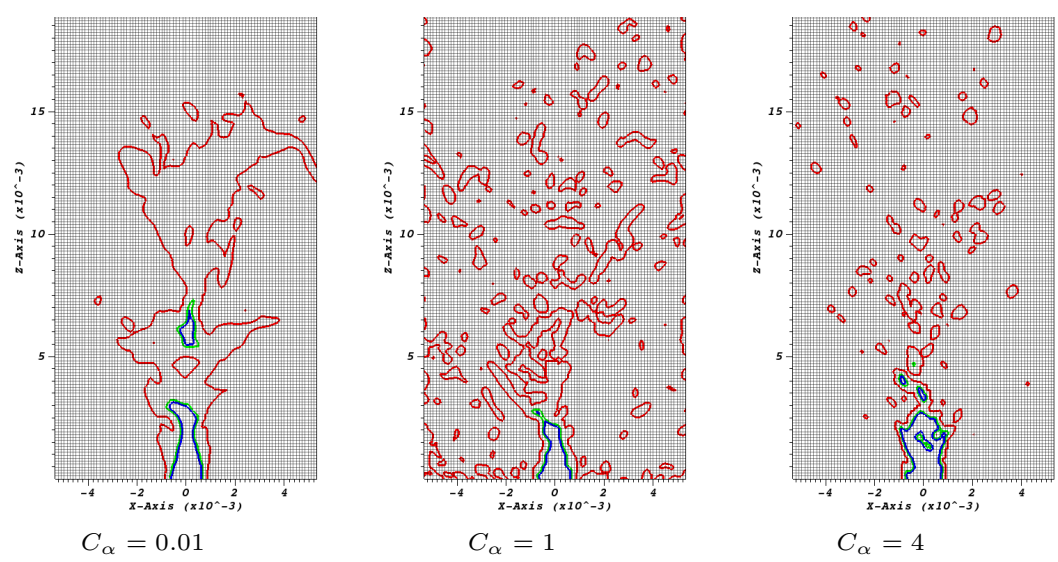

Figure 2. Iso-contours of $\alpha=0.01$ (red), 0.5 (green) and 0.7 (blue) for different compression factor.

Figures 3 and 4 show the local Weber number distributions Two different reference lengths are used: in one case the grid size, $W e_{\Delta}=\rho U^{2} \Delta / \sigma$, and in the other the local mean curvature, $W e_{\kappa}=\rho U^{2} /(\sigma \kappa)$. These choices are used to see the influence of the numerical parameters (the grid size ) and the effect of the error on the volume fraction estimate (which is translated into an error for the mean curvature), on the interface and on the breakup mechanism. As regards $W e_{\Delta}$ it is expected to be of the order of one to be resolved accurately [16] .

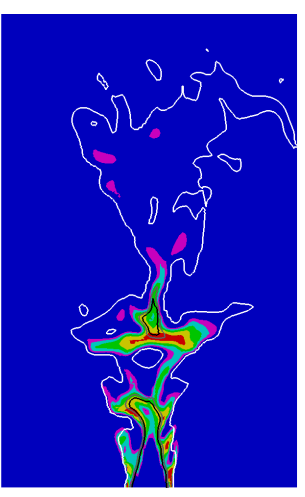

$C_{\alpha}=0.01$

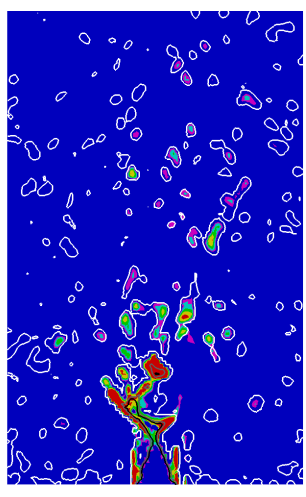

$C_{\alpha}=1$
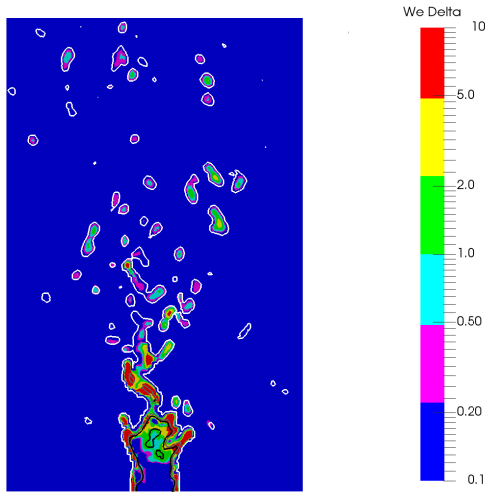

$C_{\alpha}=4$

Figure 3. Local Weber distribution, $W e_{\Delta}$, based on cell size as reference length

The more diffusive scheme, creates very smooth surfaces, with low values of density. Paradoxically, the more the diffuse the interface, the better the capillary effects are resolved. Increasing the compression constant, the local Weber $W e_{\Delta}$ increase along the ligaments and the resolution is inadequate with $W e_{\Delta}>>1$. Further downstream, some droplets are still characterised by a Weber greater than unity. However, most of the droplets are below 2 , which suggest that capillary effects are quasi-resolved.

Figure 4 shows the local Weber based on curvature. The large compression factors, show droplets with $W e_{\kappa}<10$, which suggest that they will not further break [17] as critical Weber is around 10. In the ligament region $W e_{\kappa} \sim 100$, 
which exceeds critical Weber and droplets are expected to appear. However, at $C_{\alpha}=0.01, W e_{\kappa}<10$ nearly everywhere, which prevents filaments to break into droplets. The shear layer between the gas and the air presents the strongest instabilities and large variations of $W e_{\kappa}$.

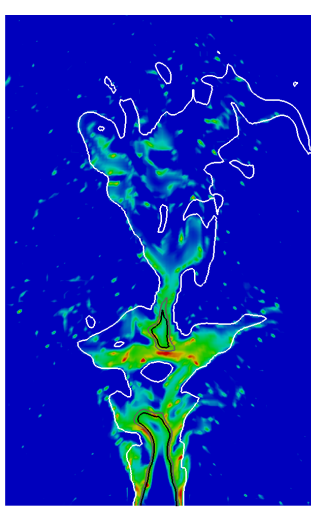

$C_{\alpha}=0.01$

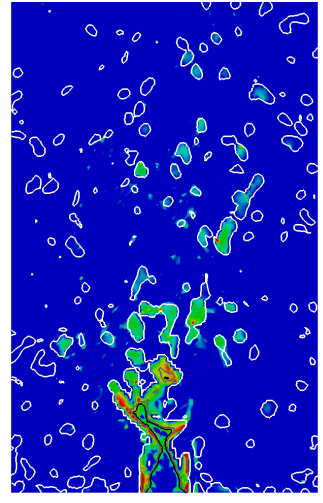

$C_{\alpha}=1$
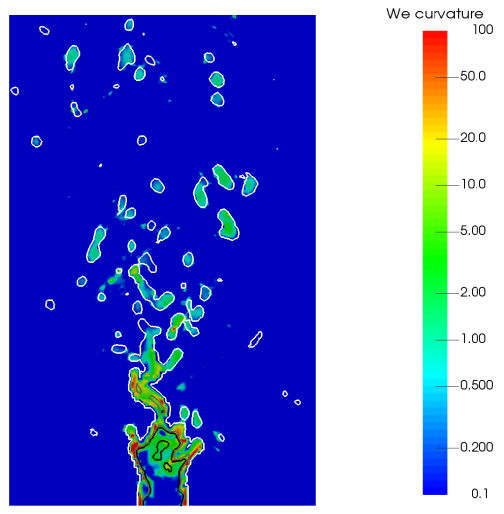

$C_{\alpha}=4$

Figure 4. Local Weber distribution, $W e_{\kappa}$, based on curvature radius as reference length

Figure 5 shows the droplet distribution of the present simulations, compared to the experimental and DNS results presented in [3]. To present fair comparison a diameter normalisation $d^{*}=\left(d-d_{\min }\right) /\left(d_{\max }-d_{\min }\right)$ has been performed. Table 2 reports the peak values $\left(d_{\text {peak }}^{*}\right)$ and mean $\left(\bar{d}^{*}\right)$ of these distributions with both the one obtained by [3]. For all the compression constant, distributions present at least a local peak close the experimental and DNS results. Due to the higher resolution, the DNS captures more and smaller droplets. Also if the the lower compression factor has a $d_{\text {peak }}^{*}$ close to the validated results, it shows slower decay than DNS and experimental, with more uniform droplets along the number, as indicated by $\bar{d}^{*}$.

The results for $C_{\alpha}=1$, show two peaks, the first one near the experimental data. However an unrealistic second peak appears at large values. The case $C_{\alpha}=4$ shows the best agreement with experimental and DNS data on the mean $d^{*}$. as well as the exponential decay at large diameter.

Table 2. Peak Values $\left(d_{\text {peak }}^{*}\right)$ and mean $\left(\bar{d}^{*}\right)$ of droplets distributions

\begin{tabular}{c|cc}
\hline & $d_{\text {peak }}^{*}$ & $\bar{d}^{*}$ \\
\hline DNS & 0.12 & 0.158 \\
Experiment & 0.04 & 0.125 \\
$C_{\alpha}=0.01$ & 0.16 & 0.408 \\
$C_{\alpha}=1$ & 0.48 & 0.409 \\
$C_{\alpha}=4$ & 0.25 & 0.208
\end{tabular}
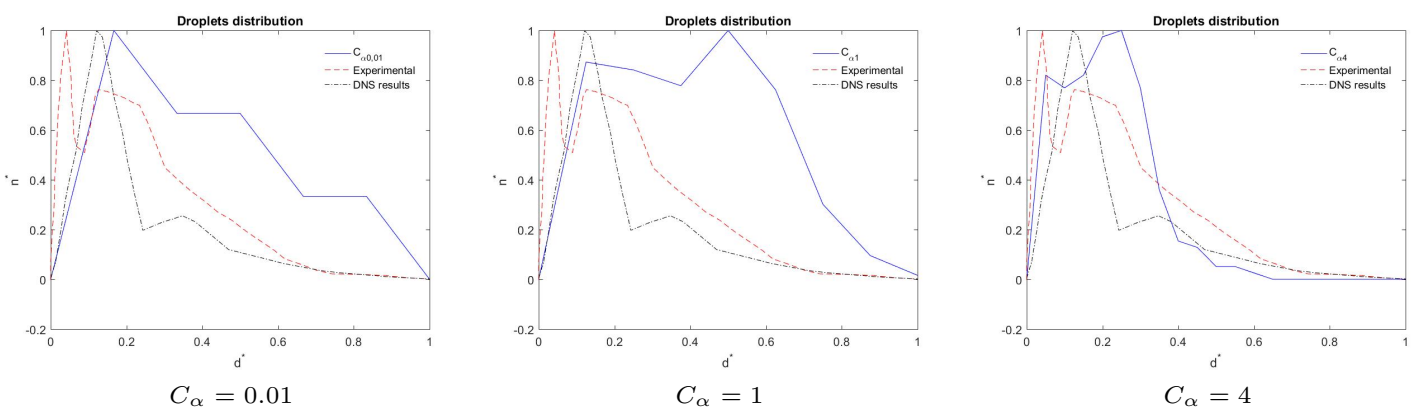

Figure 5. Normalised droplet size distribution with $d^{*}=\left(d-d_{\min }\right) /\left(d_{\max }-d_{\min }\right)$

In this sections, the same simulations set-up are proposed, but a filter process is applied to $\alpha$ in the interface region using a Laplacian filter [13], viz.

$$
\tilde{\alpha}_{P}=\frac{\sum_{f=1}^{n} \alpha_{f} S_{f}}{\sum_{f=1}^{n} S_{f}}
$$


where the subscripts $P$ indicates the cell index. The smoothed volume fraction obtained by $(13)$ is used to compute the interface mean curvature $\kappa$ and the surface normal $\mathbf{n}$.

The results show large difference. Figure 6 shows the results at low compression factor. The liquid detachment of the liquid structure from the dense core is much clearer now and consist of big structures $\sim d_{1}$ that break further downstream. However, no small droplets are present and the results are not realistic. Increasing interface compression, (Figure 7 ), there is no detachment of liquid structure and there is the formation of ligaments of liquid, which breaks producing droplets which size at least one order of magnitude smaller than liquid inlet diameter. The smoothing of the $\alpha$ marker, creates a larger spray angle and some filament-like structures.

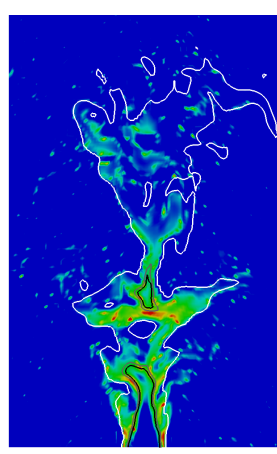

$\alpha$

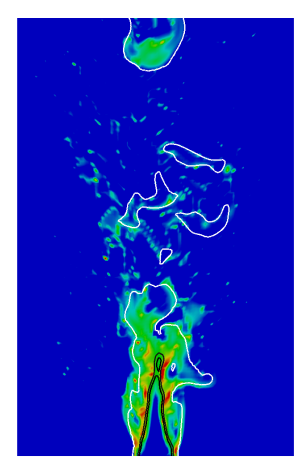

$\tilde{\alpha}$

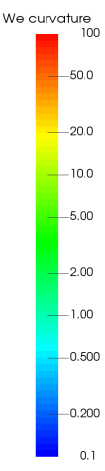

Figure 6. Local Weber distribution, $W e_{\kappa}$, based on curvature radius as reference length for $C_{\alpha}=0.01$. Original $\alpha$ (left), smooth $\tilde{\alpha}$ (right)

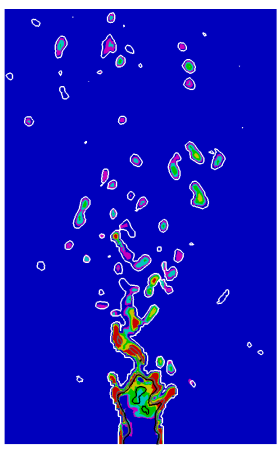

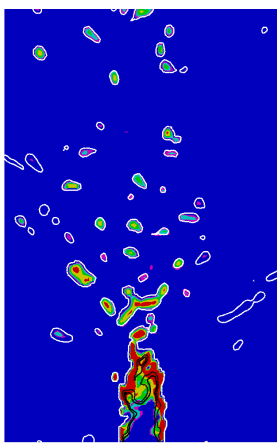

$\tilde{\alpha}$

Figure 7. Local Weber distribution, $W e_{\Delta}$, based on cell size as reference length for $C_{\alpha}=4$. Original $\alpha$ (left), smooth $\tilde{\alpha}$ (right)

Table 3. Peak Values $\left(d_{\text {peak }}^{*}\right)$ and mean $\left(\bar{d}^{*}\right)$ of droplets distributions for smooth $\tilde{\alpha}$.

\begin{tabular}{c|cc}
\hline & $d_{\text {peak }}^{*}$ & $\bar{d}^{*}$ \\
\hline DNS & 0.12 & 0.158 \\
Experiment & 0.04 & 0.125 \\
$C_{\alpha}=0.01$ & 0.4 & 0.508 \\
$C_{\alpha}=1$ & 0.55 & 0.40 \\
$C_{\alpha}=4$ & 0.19 & 0.164
\end{tabular}

Although the trend is the same as non smoothing case, looking at Table 3 the results drift from the experimental data for low and intermediate compression. For the $C_{\alpha}=4$, Figure $8 \mathrm{c}$, both $d_{\text {peak }}^{*}$ and $\bar{d}^{*}$ are close to the DNS results, showing the best agreement for the droplet distribution.

\section{Conclusions}

The simulation of an air-assisted atomiser at intermediate Weber numbers were carried out in this work. The effects of the compression factor were investigated. The description of the liquid core, as the core length or the 

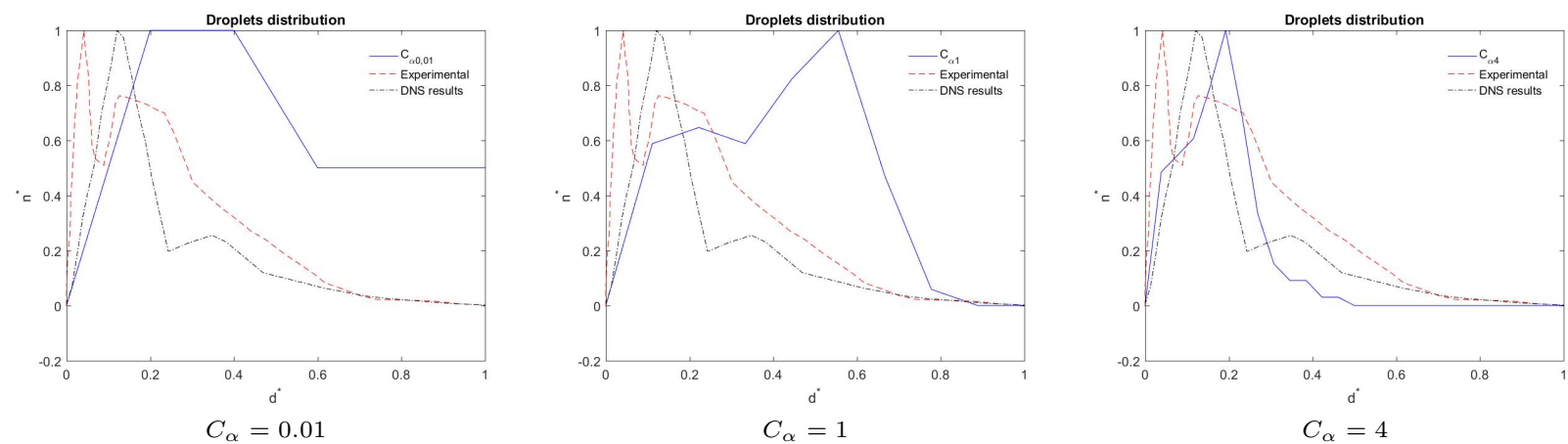

Figure 8. Normalised droplet size distribution with $d^{*}=\left(d-d_{\min }\right) /\left(d_{\max }-d_{\min }\right)$ for smooth $\tilde{\alpha}$.

interface shape, are better obtained by compression factors below unity. However downstream the injector, the low compression factors barely produced any droplets and only the largest compression factors produce similar droplet size distribution than DNS and experimental data. The results show that for the atomisation process at intermediate Weber, an unique compression factor is not useful.

At the injection a small compression factor is needed. However, downstream the injector, at the top of the dense core, more compression is needed to allow the formation of ligaments and create droplets. Increasing the compression factor augments the density, and therefore the value of the local $W e_{\Delta}$, reducing the quality of the capillary/inertial forces interaction. Smoothing the marker function, does not improve the overall results although it greatly affects the break-up shape at low compression factors, however it reduces the amount of small droplets produced. For low compression and without smoothing, the error for both the curvature and the normal interface is too large. Increasing the compression the interface is sharper improving the curvature but giving worse results for the normal vectors. Introducing the smoothing, the estimate of these interface parameters is improved, giving a better estimate for the surface force that will define the breakup and so the particle distributions. The results suggest that a curvature-based Weber, $W e_{\kappa}$, could be used as a marker for the compression factor in a form $C_{\alpha} \propto 1 / W e_{\kappa}$. Close to the nozzle, $W e_{\kappa}$ is large and therefore low compression is needed to accurately capture the propagation of instabilities. However this is reversed as the break-up approaches and more compression is needed. This will be subject of future work.

\section{Acknowledgements}

This work is part of the HAoS project, which is supported by the EU as part of the Horizon 2020 program.

\section{Nomenclature}

$\alpha \quad$ volume fraction [-]

$\tilde{\alpha} \quad$ smoothed volume fraction [-]

$\mu \quad$ dynamic viscosity $\left[\mathrm{kg} \mathrm{m}^{-1} \mathrm{~s}^{-1}\right]$

$\sigma \quad$ surface tension $\left[\mathrm{N} \mathrm{m}^{-1}\right]$

$\kappa \quad$ mean curvature $\left[\mathrm{m}^{-1}\right]$

$\rho \quad$ density $\left[\mathrm{kg} \mathrm{m}^{-3}\right]$

$p \quad$ pressure $\left[\mathrm{kg} \cdot \mathrm{m}^{-1} \mathrm{~s}^{-2}\right]$

$\mathrm{u} \quad$ velocity $\left[\mathrm{m} \mathrm{s}^{-1}\right]$

f force [N]

$\mathbf{S}_{f} \quad$ cell face area [-]

$C_{\alpha} \quad$ compression factor constant [-]

$d \quad$ droplet diameter [mm]

$n$ number of droplet [-]

\section{References}

[1] Ishi, M, 1975, "Thermo-fluid Dynamic Theory of Two-phase Flow", Eyrolles, Paris/Scientific and Medical Publications of France.

[2] Marmottant, P., and Villermaux, E., 2004, Journal of fluid mechanics, 498 , pp. 73-111.

[3] Desjardins, O., McCaslin, J., Owkes, M., Brady, P., 2013, Atomization and Sprays, 23 (11), pp. 1001-1048.

[4] Sirignano, W.A. and Mehring, C., 2000, Progress in Energy and Combustion Science, 26 (4), pp. 609-655.

[5] Eggers, J., and Villermaux, E., 2008, Reports on progress in physics, 71 (3), pp. 1-19.

[6] Hirt, C.W.; Nichols, B.D. ,1981, Journal of Computational Physics, 39 , 201-225

[7] Tryggvason, G., Scardovelli, R, Zaleski, S., 2011, "Direct numerical simulations of gas-liquid multiphase flows". 
Cambridge University Press.

[8] Brackbill, JU., Kothe, D., Zemach, C., 1992, Journal of computational physics, 100 (2), pp. 335-354.

[9] Weller, H.G., Tabor, G., Jasak, H., Fureby, C., 1998, Computer in physics, 12 (6), pp. 620-631.

[10] OpenCFD, Technical Report No. TR/HGW/02, 2005 (unpublished).

[11] Damián, S. M., 2013 Ph.D. thesis , An extended mixture model for the simultaneous treatment of short and long scale interfaces.

[12] Aboukhedr, M., Gavaises, M., Georgoulas, A., Marengo, M., Vogiatzaki, K., 27th Annual Conference on Liquid Atomization and Spray Systems .

[13] Lafaurie, B., and Nardone, C., and Scardovelli, R., and Zaleski, S., and Zanetti, G., 1994, Journal of Computational Physics, 113 (1), pp. 134-147.

[14] Aubin, J., Abadie, T. and Legendre, D., 2016 Journal of computational physics, 297, pp. 611-636.

[15] Lasheras, J. and Hopfinger, H., 2000 Annual Review of Fluid Mechanics, 32, pp. 275-308.

[16] Menard, T., Tanguy, S. and Berlemont, A., 2007, Int. J. Multiphase Flow, 33, pp 510-524

[17] Desjardins, O and Pitsch, H, 2009 J. Comp. Phys., pp. 1658-1677. 